\title{
An Optimization Model of Railway Empty Container Allocation Based on Customer Satisfaction
}

\author{
Zhiwei Zhang ${ }^{1}$, Xiaohong $\mathrm{Li}^{2, \text { a }}$ \\ ${ }^{1}$ School of Traffic and Transportation, Beijing Jiaotong University, Beijing, China \\ ${ }^{2}$ School of Traffic and Transportation, Beijing Jiaotong University, Beijing, China
}

\begin{abstract}
With the rapid development of modern corporate management, customer satisfaction evaluation has been applied to the management of many companies. Railway empty container allocation not only involves the cost of the railway transportation, but also considers the customer's requirements for time and convenience, etc. Therefore customer satisfaction evaluation also becomes very important for railway container allocation decision. However, it is not easy to properly quantify customer satisfaction, because there are many indicators that cannot be directly quantified. This paper proposes a customer satisfaction evaluation index system based on the RATER index, and adopts a comprehensive evaluation method to achieve quantitative evaluation of customer satisfaction. An empty container allocation optimization model based on customer satisfaction is established. The model can comprehensively consider the interests of both the railway and customers, and provide an effective reference for the railway transportation department to improve the transportation service and optimize empty container allocation plan.
\end{abstract}

\section{Introduction}

In the statistics of container transportation expenses of China Railway Container Transport Co., Ltd., the expenses incurred due to empty container allocation accounted for $7.97 \%$ of the total payment, while the railway empty container transportation accounted for $21.9 \%$ of the total transportation volume [1]. A scientific and reasonable railway empty container allocation plan can not only accelerate the use of railway empty containers, improve the utilization rate of railway empty containers, but also meet the customer's container demand and improve the market service effectively. Therefore, it is necessary to apply customer satisfaction evaluation to optimize the empty container allocation plan.

\section{Literature review}

Currently, there are many research efforts focused on the customer satisfaction of railway freight transportation. E. Hirata [2] mapped the quality of service dimension to a new set of service characteristics based on the latest priority of shipping companies, and discussed the key service characteristics of the container liner shipping (CLS) industry and its impact on customer satisfaction. J.T. Mentzer et al. [3] constructed the logistics service quality model for evaluating customer logistics service satisfaction based on the characteristics of logistics service quality. Y.Y. Tang et al. [4] established a evaluation index system and combined the fuzzy

\footnotetext{
a Corresponding author: xhli1@bjtu.edu.cn
}

evaluation with the service quality evaluation model to evaluate the quality of railway freight services.

Scholars also have done a lot on the empty container allocation. J.L. He et al. [5] designed the container empty container allocation route into a transportation network, and established a multi-level container logistics supply chain network (MCSCN) optimization model. E.Y.C. Wong et al. [6] proposed an empty container allocation model based on uncertain factors and made empty container deployment with better routes. T. Luo et al. [7] applied the contract coordination theory to the empty container allocation model to improve the coordination of empty container management and the initiative of each participant. Y. Myung [8] developed a solution algorithm for the two models in literature 7 and proved that the network flow algorithm could also solve the other two models. S.T. Choong et al. [9] established an empty container allocation integer programming model under multimodal transport by analysing the status of empty container allocation in the Great Lakes region of the United States.

\section{Customer satisfaction evaluation}

One of the foundations of enterprise development is customer satisfaction. In the production and operation, the company should firmly establish the business concept of "customer-centric", and constantly improve product quality to ensure the company's strong market competitiveness. 
We use the AHP-fuzzy comprehensive evaluation method to calculate the customer satisfaction. Firstly, we establish a customer satisfaction index system for empty container allocation through the RATRA index. Then, we employ the 1-5 scale method to calculate the index weight. Finally, we adopt the evaluation principle with the highest degree of membership in the AHP-fuzzy evaluation method to analyze the evaluation coefficient of customer satisfaction.

\subsection{Customer satisfaction evaluation index system}

Scientific, reasonable and feasible evaluation indicators are the premise for correctly evaluating customer satisfaction, and also an important basis for establishing an empty container allocation model. The RATER index measures the quality of customer service by the US-based authoritative customer service research organization, Tribune Company, It represents the five major factors that measure the quality of business services and comprehensively demonstrates the partnership between the company and its users. It can fully reflect the customer's satisfaction with the railway transportation department. The scientific application of the RATER index can enhance the competitiveness of the railway market, and be an important method for user management in the railway transportation. This paper embodies the RATER index and obtains an index system that meets the actual situation of railway empty container deployment. The indicator system consists of five first-level indicators and nine second-level indicators. The specific indicator system is shown in Figure 1.

\subsection{Customer satisfaction index weight}

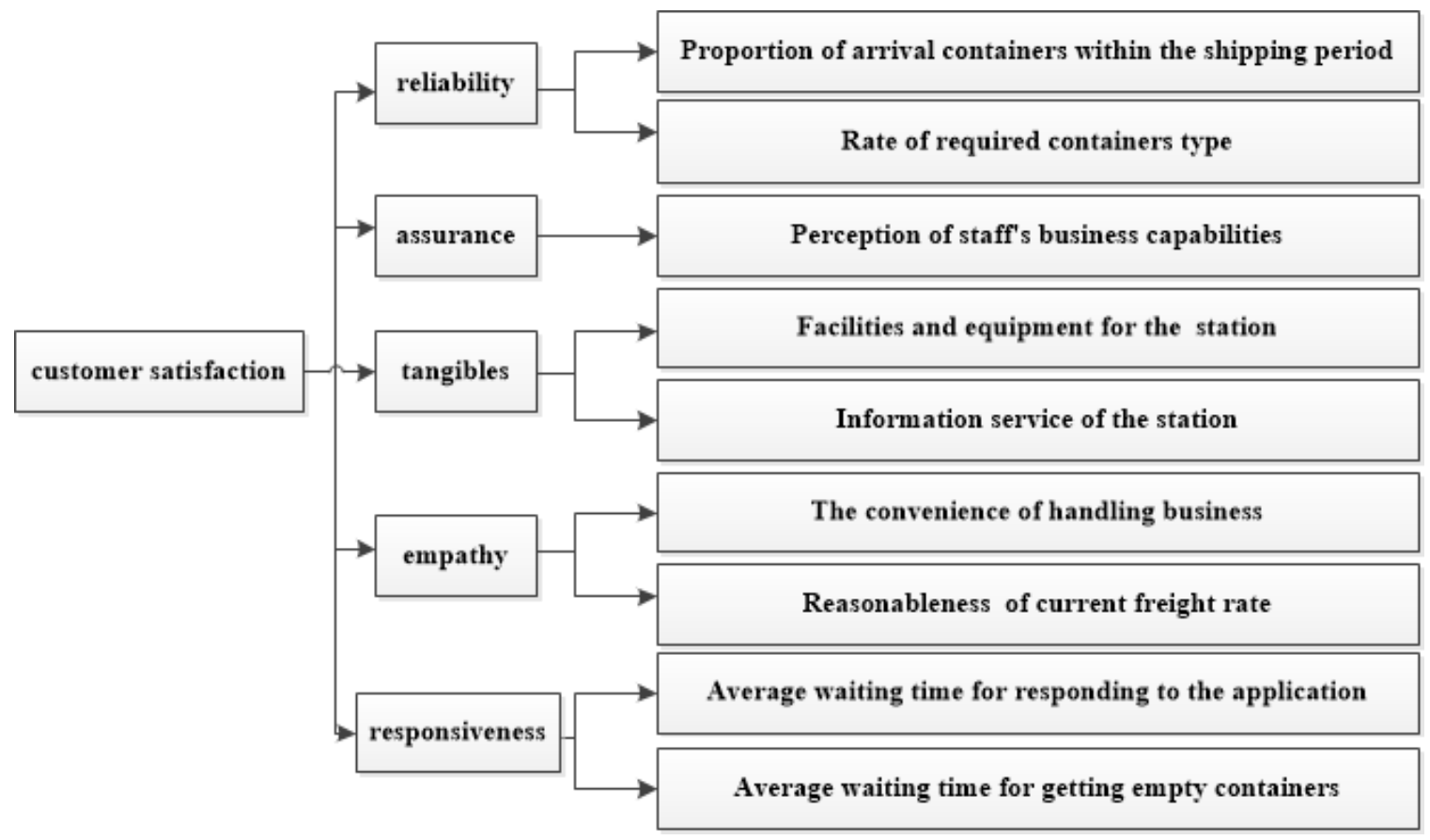

Figure 1. Customer satisfaction evaluation index. 


\subsection{AHP-fuzzy comprehensive evaluation of customer satisfaction}

We assume that the number of the supplying station participating in the evaluation are $i(i=1,2,3,4 \ldots ., \mathrm{m})$, the number of the demanding station participating in the evaluation are $j(j=1,2,3,4, \ldots, n)$. Customers participating in the survey rate evaluation index for each station according to relevant experience and comment set. We collect the questionnaire and calculate the fuzzy comprehensive evaluation coefficient $\mu$ of each station according to the AHP-fuzzy comprehensive evaluation method. We define:

$$
\mu_{i j}=\mu_{i} \cdot \mu_{j}
$$

Eq. (2) means customer satisfaction index from the supplying station $i$ to the demanding station $j$ in the empty container allocation system.

\section{Model formulation}

\subsection{Assumptions}

In order to perform the quantitative analysis, the paper give the following assumptions:

(1)The supply-demand relationship between container stations is fixed for a certain period. It is a static system and does not consider dynamic changes caused by uncertain factors.

(2) It is possible to carry out the substitution of the container type and the vehicle type.

(3) There is no empty container convection between the two stations.

\subsection{Declaration of parameter variable}

List the variables and parameters used in this article as follow.

$x_{i j}^{p q}$ denotes the number of empty $p$-containers provided by station $i$ to replace the empty $q$-containers needed by station $j . y_{i j}^{g h}$ denotes the number of $g$ vehicles to replace $h$-vehicles from station $i$ to station $j$. I denotes the set of supply stations. J denotes the set of demand stations. $D_{j}^{q}(j=1,2,3,4, \ldots, n)$ denotes the quantity of empty $q$-containers needed by the station $j$. $S_{i}^{p}(i=1,2,3,4, \ldots, m)$ denotes the quantity of empty $p$-containers provided by the station $i . V e_{i}^{g}$ denotes the quantity of $g$-vehicles provided by the station $i . c_{i j}^{p}$ denotes the cost of transporting one unit of empty $p$ containers. $c_{i j}^{\prime p}$ denotes the generalized cost of transporting one unit of empty p-containers. $c_{i j}^{g h}$ denotes the additional cost of replacing the $h$-vehicle by the $g$ vehicle, When $g=h, c_{i j}^{g h}=0 . c_{i j}^{\prime g h}$ denotes the additional generalized cost of replacing the $h$-vehicle by the $g$ - vehicle, When $g=h, c_{i j}^{\prime g h}=0 . \alpha_{j}^{p q}$ denotes whether the empty $q$-container needed for the station $j$ is replaced by an empty $p$-container, which can be replaced by $\alpha_{j}^{p q}=1$, otherwise, $\alpha_{j}^{p q}=0 . \varphi_{i}^{g h}$ denotes whether the $h$-vehicle is replaced by a $g$-vehicle provided for the station $i$, which can be replaced by $\varphi_{i}^{g h}=1$, otherwise, $\varphi_{i}^{g h}=0 . \beta_{j}^{p q}$ denotes the penalty factor when the $q$-container needed by the station $j$ is replaced by a $p$-container, when $p=q$, $\beta_{j}^{p q}=1$. $\omega$ denotes the multiple of the number between vehicles and containers. $\mathrm{Cap}_{i j}$ denotes the maximum capacity of $\operatorname{arc}(i, j) . u_{j}$ denotes the importance coefficient of station $j . u_{i}$ denotes the importance coefficient of station $i . \mu_{i j}$ denotes the customer satisfaction coefficient between station $i$ and station $j$.

\subsection{Objective function}

The goal of the optimization model is to minimize the generalized cost required for allocation. The total generalized cost consists of two parts. The first part is the generalized allocation cost generated by empty container transportation, the second part is the generalized substitute cost generated by vehicle substitution.

$$
\begin{gathered}
\min Z=\sum_{i=1}^{m} \sum_{j=1}^{n} \sum_{p} \sum_{q} c_{i j}^{\prime p} \cdot x_{i j}^{p q} \cdot \beta_{j}^{p q} \\
+\sum_{i=1}^{m} \sum_{j=1}^{n} \sum_{g} \sum_{h} c_{i j}^{g h} \cdot y_{i j}^{g h}
\end{gathered}
$$

\subsection{Constraints}

$$
\begin{aligned}
& c_{i j}^{\prime p}=\frac{c_{i j}^{p}}{u_{i} u_{j} \mu_{i j}} \quad \forall i, j \\
& c_{i j}^{\prime g h}=\frac{c_{i j}^{g h}}{u_{i} u_{j} \mu_{i j}} \quad \forall i, j \\
& \sum_{i=1}^{m} \sum_{p} x_{i j}^{p q} \cdot \alpha_{j}^{p q}=D_{j}^{q} \quad \forall j, q \\
& \sum_{j=1}^{n} \sum_{q} x_{i j}^{p q} \cdot \alpha_{j}^{p q} \leq S_{i}^{p} \quad \forall i, p \\
& \sum_{j=1}^{n} \sum_{h} \varphi_{i}^{g h} \cdot y_{i j}^{g h} \leq V e_{i}^{g} \quad \forall i, g \\
& \sum_{g} \sum_{h} y_{i j}^{g h}=\sum_{p} \sum_{q} \frac{x_{i j}^{p q}}{\omega} \quad \forall i, j \\
& \sum_{p} \sum_{q} \frac{x_{i j}^{p q}}{\omega} \leq C a p_{i j} \quad \forall i, j \\
& x_{i j}^{p q} \geq 0, y_{i j}^{g h} \geq 0 \quad
\end{aligned}
$$

and integer $\forall i, j, p, q, g, h$ (10)

Eq. (3)-(4) define the quantitative relationship between empty container allocation cost, vehicle substitute cost and generalized cost; Eq. (5) ensures that 
the number of empty containers that arrive at station $j$ is the same as the demand for empty containers at that site; Constraint (6) guarantees that the total number of empty containers moved from station $i$ is not more than the empty containers provided by that site; Constraint (7) assures that station $i$ has enough vehicles to transport empty containers; Eq. (8) defines that the sum of vehicles corresponds with the total containers from station $i$ to station $j$; Constraint (9) denotes the limitation of line capacity; $x_{i j}^{p q}$ and $y_{i j}^{g h}$ are decision variables and nonnegative integers.

\subsection{Solution}

As there is a linear relation between objective function, constraints and decision variables, this is an integer linear programming model. The most common method for solving integer programming problems is the branch and bound method. The branch and bound method, whose basic idea is to enumerate all feasible solutions which means to divide the solution space successively, uses a 'divide and rule' strategy for solving integer programming problems. Therefore, we can solve this integer linear programming model by applying LINGO11.0, which is widely used.

\section{Numerical example}

In order to verify the validity and rationality of the model, we select eight container-providing stations and seven container-demanding stations, and take vehicle and container-type substitution into account in the study. In this example, we assume that the line capacity meets the transportation need and the importance coefficient of all stations is equal to 1 . The container set is $\{20$-foot container, 40 -foot container $\}=\{T, F\}$, the container transport vehicle set is \{ gondola, container flat car $\}=\{C, X\}$, the container substitution judgment parameter matrix is $\left\{\alpha_{j}^{T T}, \alpha_{j}^{T F} ; \alpha_{j}^{F T}, \alpha_{j}^{F F}\right\}=\{1,0 ; 1,1\}$, $j=1,2, \ldots 7$, The vehicle substitute judgment parameter matrix is $\left\{\varphi_{i}^{C C}, \varphi_{i}^{C X} ; \varphi_{i}^{X C}, \varphi_{i}^{X X}\right\}=\{0,1 ; 0,1\}, i=1$, $2, \ldots 8$, The container substitution penalty factor matrix is $\left\{\beta_{j}^{T T}, \beta_{j}^{T F} ; \beta_{j}^{F T}, \beta_{j}^{F F}\right\}=\{1, \infty ; 1.21,1\}, j=1,2, \ldots 7$, The vehicle substitute cost matrix is $\left\{c_{i j}^{C C}, c_{i j}^{C X} ; c_{i j}^{X C}\right.$, $\left.c_{i j}^{X X}\right\}=\{\infty, 50 ; \infty, 0\}, i=1,2, \ldots 8, j=1,2, \ldots$ 7. The allocation cost and quantity of supply and demand of empty containers are shown in Table 2 . The number of vehicles for the container-providing station is shown in Table 3. Considering the assessment of experts and customers, we calculate a first-level indicator weight set $W=(0.3,0.13,0.17,0.2,0.2)$, The customer satisfaction evaluation coefficient set of the container-providing stations is $\left(S_{1}, S_{2}, S_{3}, S_{4}, S_{5}, S_{6}, S_{7}, S_{8}\right)=(0.42,0.56$, $0.38,0.41,0.53,0.43,0.53,0.50)$, The customer satisfaction evaluation coefficient set of the containerdemanding stations is $\left(D_{1}, D_{2}, D_{3}, D_{4}, D_{5}, D_{6}, D_{7}\right)$ $=(0.59,0.34,0.51,0.32,0.58,0.43,0.49)$.

Table 3. The number of vehicles for the container-providing station.

\begin{tabular}{|c|c|c|c|c|c|c|c|c|}
\hline station & $S_{1}$ & $S_{2}$ & $S_{3}$ & $S_{4}$ & $S_{5}$ & $S_{6}$ & $S_{7}$ & $S_{8}$ \\
\hline gondola & 50 & 15 & 51 & 20 & 40 & 20 & 30 & 10 \\
\hline $\begin{array}{c}\text { container flat } \\
\text { car }\end{array}$ & 100 & 70 & 50 & 85 & 99 & 76 & 95 & 50 \\
\hline
\end{tabular}

We solve the empty container allocation optimization model based on customer satisfaction through LINGO11.0 software. The finishing result is shown in Table 4 and Table 5.

Table 2. The allocation cost and quantity of supply and demand.

\begin{tabular}{|c|c|c|c|c|c|c|c|c|c|}
\hline \multicolumn{9}{|c|}{ Allocation cost (yuan/TEU) } & \multirow[b]{2}{*}{ Supply/TEU } \\
\hline & $\begin{array}{c}\text { type of } \\
\text { container }\end{array}$ & $D_{1}$ & $D_{2}$ & $D_{3}$ & $D_{4}$ & $D_{5}$ & $D_{6}$ & $D_{7}$ & \\
\hline \multirow{2}{*}{$S_{1}$} & $T$ & 65 & 108 & 127 & 217 & 266 & 176 & 221 & 100 \\
\hline & $F$ & 74 & 120 & 139 & 235 & 286 & 191 & 239 & 100 \\
\hline \multirow{2}{*}{$S_{2}$} & $T$ & 62 & 65 & 95 & 165 & 214 & 133 & 169 & 50 \\
\hline & $F$ & 71 & 75 & 107 & 180 & 231 & 146 & 184 & 60 \\
\hline \multirow{2}{*}{$S_{3}$} & $T$ & 137 & 94 & 174 & 109 & 212 & 51 & 120 & 40 \\
\hline & $F$ & 150 & 105 & 189 & 120 & 229 & 59 & 132 & 81 \\
\hline \multirow{2}{*}{$S_{4}$} & $T$ & 168 & 125 & 203 & 163 & 180 & 57 & 88 & 56 \\
\hline & $F$ & 183 & 138 & 220 & 178 & 196 & 66 & 99 & 77 \\
\hline \multirow{2}{*}{$S_{5}$} & $T$ & 286 & 269 & 322 & 186 & 235 & 231 & 186 & 78 \\
\hline & $F$ & 307 & 289 & 346 & 202 & 254 & 249 & 202 & 100 \\
\hline \multirow{2}{*}{$S_{6}$} & $T$ & 173 & 145 & 210 & 119 & 167 & 77 & 71 & 40 \\
\hline & $F$ & 189 & 158 & 227 & 131 & 182 & 87 & 81 & 56 \\
\hline \multirow{2}{*}{$S_{7}$} & $T$ & 252 & 219 & 289 & 107 & 155 & 151 & 137 & 66 \\
\hline & $F$ & 271 & 237 & 310 & 118 & 170 & 165 & 150 & 90 \\
\hline \multirow{2}{*}{$S_{8}$} & $T$ & 121 & 119 & 157 & 108 & 157 & 123 & 121 & 26 \\
\hline & $F$ & 133 & 132 & 172 & 120 & 171 & 135 & 134 & 35 \\
\hline \multirow{2}{*}{ Demand/TEU } & $T$ & 80 & 70 & 80 & 110 & 100 & 72 & 40 & - \\
\hline & $\mathrm{F}$ & 15 & 45 & 50 & 80 & 70 & 50 & 24 & - \\
\hline
\end{tabular}


Table 4. Empty container allocation plan considering customer satisfaction.

\begin{tabular}{|c|c|c|c|c|c|c|c|c|}
\hline & $\begin{array}{c}\text { type of } \\
\text { container }\end{array}$ & $D_{1}$ & $D_{2}$ & $D_{3}$ & $D_{4}$ & $D_{5}$ & $D_{6}$ & $D_{7}$ \\
\hline \multirow{2}{*}{$S_{1}$} & $T$ & 12 & - & 80 & - & 8 & - & - \\
\hline & $F$ & 24 & - & 18 & - & 8 & - & - \\
\hline \multirow{2}{*}{$S_{2}$} & $T$ & 24 & 50 & - & - & & - & - \\
\hline & $F$ & - & 36 & - & - & - & - & - \\
\hline \multirow{2}{*}{$S_{3}$} & $T$ & - & 8 & - & 32 & - & 9 & - \\
\hline & $F$ & - & 21 & - & & - & - & - \\
\hline \multirow{2}{*}{$S_{4}$} & $T$ & - & - & - & - & - & 56 & - \\
\hline & $F$ & - & - & - & - & - & 57 & - \\
\hline \multirow[b]{2}{*}{$S_{5}$} & $T$ & - & - & - & 78 & - & - & - \\
\hline & $F$ & - & - & - & 52 & - & - & - \\
\hline \multirow{2}{*}{$S_{6}$} & $T$ & - & - & 32 & - & - & - & 40 \\
\hline & $F$ & - & - & - & - & - & - & 24 \\
\hline \multirow{2}{*}{$S_{7}$} & $T$ & - & - & - & 28 & 66 & - & - \\
\hline & $F$ & - & - & - & - & 62 & - & - \\
\hline \multirow{2}{*}{$S_{8}$} & $T$ & 35 & - & - & - & 26 & - & - \\
\hline & $F$ & - & - & - & - & - & - & - \\
\hline
\end{tabular}

Table 5. Vehicle matching plan considering customer satisfaction.

\begin{tabular}{|c|c|c|c|c|c|c|c|c|}
\hline & $\begin{array}{l}\text { type of } \\
\text { vehicle }\end{array}$ & $D_{1}$ & $D_{2}$ & $D_{3}$ & $D_{4}$ & $D_{5}$ & $D_{6}$ & $D_{7}$ \\
\hline \multirow{2}{*}{$S_{1}$} & $C$ & - & - & - & - & - & - & - \\
\hline & $X$ & 30 & - & 58 & - & 12 & - & - \\
\hline \multirow{2}{*}{$S_{2}$} & $C$ & 15 & - & - & - & - & - & - \\
\hline & $X$ & 19 & 61 & - & - & - & - & - \\
\hline \multirow{2}{*}{$S_{3}$} & $C$ & - & - & - & - & - & - & - \\
\hline & $X$ & - & 25 & - & 16 & - & 9 & - \\
\hline \multirow{2}{*}{$S_{4}$} & $C$ & - & - & - & - & - & - & - \\
\hline & $X$ & - & - & - & - & - & 85 & - \\
\hline \multirow{2}{*}{$S_{5}$} & $C$ & - & - & - & - & - & - & - \\
\hline & $X$ & - & - & - & 91 & - & - & - \\
\hline \multirow{2}{*}{$S_{6}$} & $C$ & - & - & - & - & - & - & - \\
\hline & $X$ & - & - & 32 & - & - & - & 44 \\
\hline \multirow{2}{*}{$S_{7}$} & $C$ & - & - & - & 28 & - & - & - \\
\hline & $X$ & - & - & - & - & 95 & - & - \\
\hline \multirow{2}{*}{$S_{8}$} & $C$ & - & - & - & - & - & - & - \\
\hline & $X$ & 35 & - & - & - & 13 & - & - \\
\hline
\end{tabular}

When the customer satisfaction is not considered, we minimize the actual cost as the optimization goal for empty container allocation. The plan obtained by LINGO 11.0 is shown in Table 6 and Table 7.

Overall, the generalized cost of the model considering customer satisfaction is 28,845 yuan less than that of the ordinary model, which is $5.6 \%$ lower than the latter. The actual total cost of the allocation model based on customer satisfaction is $4.3 \%$ higher than the ordinary model, which is the price of considering the interest of the customer. The costs of the two models are shown in Figure 2. The empty container allocation model based on customer satisfaction can consider the interests of both the railway department and the customer, and strive for a balance between the two by a small increase of actual cost.
Table 6. Empty container allocation plan without considering customer satisfaction.

\begin{tabular}{|c|c|c|c|c|c|c|c|c|}
\hline & $\begin{array}{l}\text { type of } \\
\text { container }\end{array}$ & $D_{1}$ & $D_{2}$ & $D_{3}$ & $D_{4}$ & $D_{5}$ & $D_{6}$ & $D_{7}$ \\
\hline \multirow{2}{*}{$S_{1}$} & $T$ & 46 & - & 54 & - & - & - & - \\
\hline & $F$ & 15 & - & 35 & - & - & - & - \\
\hline \multirow{2}{*}{$S_{2}$} & $T$ & - & 24 & 26 & - & - & - & - \\
\hline & $F$ & - & 45 & 15 & - & - & - & - \\
\hline \multirow[b]{2}{*}{$S_{3}$} & $T$ & - & 46 & - & 40 & - & - & - \\
\hline & $F$ & - & - & - & 35 & - & - & - \\
\hline \multirow{2}{*}{$S_{4}$} & $T$ & - & - & - & - & 11 & 56 & - \\
\hline & $F$ & - & - & - & - & - & 66 & - \\
\hline \multirow{2}{*}{$S_{5}$} & $T$ & - & - & - & 44 & 16 & - & - \\
\hline & $F$ & - & - & - & - & - & - & - \\
\hline \multirow[b]{2}{*}{$S_{6}$} & $T$ & - & - & - & - & 32 & - & 40 \\
\hline & $F$ & - & - & - & - & - & - & 24 \\
\hline \multirow{2}{*}{$S_{7}$} & $T$ & - & - & - & 45 & 66 & - & - \\
\hline & $F$ & - & - & - & - & 45 & - & - \\
\hline \multirow{2}{*}{$S_{8}$} & $T$ & 34 & - & - & 26 & - & - & - \\
\hline & $F$ & - & - & - & - & - & - & \\
\hline
\end{tabular}

Table 7. Vehicle matching plan without considering customer satisfaction.

\begin{tabular}{|c|c|c|c|c|c|c|c|c|}
\hline & $\begin{array}{l}\text { type of } \\
\text { vehicle }\end{array}$ & $D_{1}$ & $D_{2}$ & $D_{3}$ & $D_{4}$ & $D_{5}$ & $D_{6}$ & $D_{7}$ \\
\hline \multirow{2}{*}{$S_{1}$} & $C$ & - & - & - & - & - & - & - \\
\hline & $X$ & 38 & - & 62 & - & 12 & - & - \\
\hline \multirow{2}{*}{$S_{2}$} & $C$ & - & 15 & - & 5 & - & - & - \\
\hline & $X$ & - & 42 & 28 & 50 & - & - & - \\
\hline \multirow{2}{*}{$S_{3}$} & $C$ & - & - & - & - & - & - & - \\
\hline & $X$ & - & 46 & - & 16 & - & - & - \\
\hline \multirow[b]{2}{*}{$S_{4}$} & $C$ & - & - & - & - & 11 & 9 & - \\
\hline & $X$ & - & - & - & - & - & 85 & - \\
\hline \multirow{2}{*}{$S_{5}$} & $C$ & - & - & - & - & - & - & - \\
\hline & $X$ & - & - & - & 22 & 8 & - & - \\
\hline \multirow[b]{2}{*}{$S_{6}$} & C & - & - & - & - & - & - & - \\
\hline & $X$ & - & - & 32 & - & 32 & - & 44 \\
\hline \multirow{2}{*}{$S_{7}$} & $C$ & - & - & - & 28 & - & - & - \\
\hline & $X$ & - & - & - & 17 & 78 & - & - \\
\hline \multirow{2}{*}{$S_{8}$} & $C$ & - & - & - & - & - & - & - \\
\hline & $X$ & 34 & - & - & 13 & - & - & - \\
\hline
\end{tabular}

m without customer satisfaction = with customer satisfaction

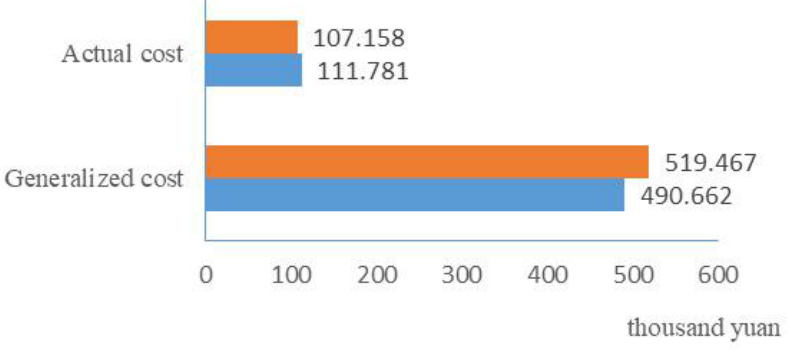

Figure 2. Comparison of costs between two models.

From a part perspective, the customer satisfaction evaluation result of $S_{2}-D_{1}$ is better than that of $S_{1}-D_{1}$. Based on the customer satisfaction, the generalized cost 
of the empty container for station $D_{1}$ decreased by $9.3 \%$, and the actual cost increased by $4.9 \%$. The balance between the empty container allocation cost and customer satisfaction is realized by increasing a small part of the actual cost.

In addition, through the AHP-fuzzy comprehensive evaluation method to quantitatively study customer satisfaction, we can find out the problems existing in the empty container allocation system, and provide the improvement direction and optimization measures for the operation and management of container transportation. For example, the customer satisfaction evaluation results of the station $S_{1}$ are (reliability, assurance, tangibles, empathy, responsiveness $)=(0.08,0.13,0.42,0.24,0.13)$, Therefore, the improvement measures for the empty container deployment service are proposed:

(1) Optimize the empty container allocation transportation organization, ensure that the empty container is transported to the time limit.

(2) Strengthen the concept of service, and improve the transportation service standards.

(3) Further optimize the information service, quickly and conveniently serve customers, and shorten the waiting time to apply for empty containers.

\section{Conclusion}

This paper proposes an optimization model for the empty container allocation based on customer satisfaction, which provides an effective reference for the railway to improve the transportation service and optimize empty container allocation plan. Firstly, we establish a customer satisfaction index system based on the RATER index. Secondly, we effectively quantify customer satisfaction via AHP-fuzzy comprehensive evaluation method. Finally, the optimization model converts actual costs into generalized costs through customer satisfaction evaluation coefficient, and comprehensively consider the interests of both the railway and the customer to optimize empty container allocation plan. This model gives out the importance coefficient of stations, and the example assumes those are equal. However, the importance coefficients are possibly different in practice. How to quantitatively study the weight of stations is the direction that need further research and discussion in the future.

\section{References}

1. C. Zhong, J. Feng, Analysis of Transportation Cost of Railway Empty Container. J. Railway Transport and Economy. 3, 41-43(2005)

2. E. Hirata, Service characteristics and customer satisfaction in the container liner shipping industry. J. The Asian Journal of Shipping and Logistics. 35(1), 24-29(2019)

3. J.T. Mentzer, D.J. Flint, G.T.M. Hult, Logistics Service Quality as a Segment—customized Process. J. The Journal of Marketing. 4, 82-101( 2001)

4. Y.Y. Tang, W.W. Li, Customer Satisfaction Evaluation of Railway Freight Service Quality Based on SERVQUAL. J. Chinese Railways. 8, 15-19(2016)

5. J.L. He, Y.F. Huagn, D.F. Chang, Simulation-based Heuristic Method for Container Supply Chain Network Optimization. J. Advanced Engineering Informatics. 29(3), 339-354 (2015)

6. E.Y.C. Wong, A.H. Tai, M. Raman, A maritime container repositioning yield-based optimization model with uncertain upsurge demand. $\mathrm{J}$. Transportation Research Part E: Logistics and Transportation Review. 82, 147-161 (2015)

7. T. Luo, D. Chang, Empty container repositioning strategy in intermodal transport with demand switching. J. Advanced Engineering Informatics. 40, 1-13 (2019)

8. Y. Myung, Efficient solution methods for the integer programming models of relocating empty containers in the hinterland transportation network. J. Transportation Research Part E: Logistics and Transportation Review. 108, 52-59 (2017)

9. S.T. Choong, H.C. MICHAEL, E. Kutanogul, Empty container management for intermodal transportation networks. J. Transportation Research Part E: Logistics and Transportation Review. 38(11), 423$438(2002)$ 